UC-20f

lsoued: Septamber 1984

$L A--10178-1.1 S$

DE85 002340

\title{
Resistive Evolution of General Plasma Configurations
}

\author{
Guthrie Maller
}

\section{DISCLAIMER}

This report was prepared as an account of work sponsored by an agency of the United States Government. Neither the United States Government nor any agency thereof, nor any of their employees, makes any warranty, express or implied, or assumes any legal liability or responsibility for the accuracy, completeness, or usefulness of any information, apparatus, product, or process disclosed, or represents that its use would not infringe privately owned right: Reference herein to any specific commercial product, process, or service by trade name, trademark, manufacturer, or otherwise does not necessarily constitute or imply its endorsement, recommendation, or favoring by the United States Government or any agency thereof. The views and opinions of authors expressed herein do not necessarily state or reflect those of the United States Government or any agency thereof.

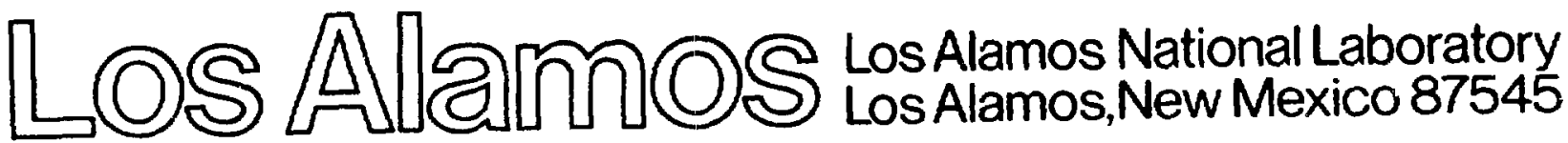




\title{
RESISTIVE EVOLUTION OF GENERAL PLASMA CONFIGURATIONS
}

by

Guthrie Miller

\begin{abstract}
The resistive evolution through equilibrium states of general plasma configurations with closed magnetic field lines is described. Cases where the magnetic field forms magnetic surfaces and where the magnetic field is ergodic are treated. In the former case, a simple equation for the rate of chenge of rotationel transform at fixed values of toroidal flux is obtained, as is already known. In the latter case the evolution of the equilibrium is naturally described in terms of the magnetic helicity by use of the formalism of relaxed states introduced by J. B. Taylor. The equation for rate of change of magnetic helicity is shown to be a general law of resistive evoiution, implying the former equation for rotational transform in the case of magnetic surfaces. In principle, the resistive evolution model provides a complete description of global long-time-scale plasma behavior in the limit where the plasma density vanishes. In this limit, the magnetohydrodynamic description of a plasma is not practical because of the vanishing of the inertial time scale.
\end{abstract}

\section{I . INTRODUCTION}

We consider the fluid description of a plasma-magnetic field system with the Ohm's law:

$$
-\frac{\partial \Lambda}{\partial t}-\nabla \Phi+v \times B=\eta j
$$

where $A$ is the vector potential, is the scalar potential, $\nabla$ is the plasma flow velocity, $B=\nabla \times A$ is the magnetic field, $j=\nabla \times B$ is the current, and $\eta$ is the plasma resistivity. The structure of Eq. (1), while mathematically 
peculiar, seens to be natural in physics in that an analogous form also applies to a Navier-Stokes lluid."

We postulate that ihe plasma is at every instant of time in mechanical equilibrium so that, in addition to Eq. (1), the following equations define the mathematical model under consideration:

$$
\begin{aligned}
& \nabla_{P}=j \times B \\
& j=\nabla \times B \\
& B=\nabla \times A
\end{aligned}
$$

where $p$ is the plasma pressure. The complete neglect of the acceleration terms in the equation of motion to obtain Eq. (2a) corresponds to the limit $p$ $\rightarrow 0$, where $\rho$ is the plasma density, or the dimensionless 1 imit $\rho \eta^{2} /\left(B^{2} a^{2}\right) \rightarrow 0$, where $a$ is a characteristic scale length. In this limit, the straightforward numerical solution of the magnetohydrodynamic equations is not practical because the numerical time step is limited by numerical stability to be vanishingly small. This limit corresponds to a parameter regime of great interest and a regime for which there is not at present a good method for global long-time-scale plasma description.

The resistive evolution model has been considered by many authors ${ }^{1-18}$ for various special cases. The evolution of three-dimensional configurations with magnetic surfaces has been considered by Grad,19,20 using the method of flux-surface averaging, and by Pereversev et. al 21 and Shafranov, 22 using a method simjlar to that used here.

"The Navier-Stokes equation can be written as

$$
-\frac{\partial v}{\partial t}-\nabla\left(\frac{p}{\rho}+\frac{v^{2}}{2}\right)+v \times \omega=\nu \nabla \times \omega,
$$

where $\nabla, p, p, \omega$, and $\nu$ are, respectively, fluid velocity, pressure, density, vorticity $(\omega=\nabla \times v)$, and kinematic viscosity. 
In magnetohydrodynamics, the velocity is advanced in time using the equation of motion. In equilibrium evolution, however, the velocity is determined indirectly in a nonobvious way by the constraints imposed by Eqs. (1) and (2). The key property of the equilibrium evolution model is that magnetic field evolution proceeds independently of the velocity and scalar potential so that the velocity and scalar potential entirely drop out of the governing equations. To understand this we first consider a general implication of Eq. (1) if closed lines of B or v exist; namely.

$$
-\oint \frac{\partial A}{\partial t} \cdot d l=\oint \eta j \cdot d l
$$

where the line integral is taken around a closed $B$ or $v$ line. We consider only the case where all magnetic field lines are closed. By integrating Eq. (1) around a closed magnetic field line we obtain Eq. (3), or al ternatively,

$$
-\frac{d}{d t} A \cdot d \ell=\eta j \cdot d \ell
$$

where the field line can be considered to move with arbitrary velocity or can be considered stationary. Equation (4) supplies one equation for every closed magnetic field line. This is just sufficient information to specify the evolution of the plasna equilibrium because of the structure of Eqs. (2), as vill be discussed subsequently in detail.

The velocity and scalar potential have been eliminated from the problem and are yet to be determined. This is done as follows. ${ }^{23}$ By dotting Eq. (1) with $B$ we obtain

$$
-B \cdot \nabla \cdot=\left(\eta j+\frac{\partial A}{\partial t}\right) \cdot B
$$


To specify $\$$, we must choose a gauge, and having done so, $A$ and $\partial A / \partial t$ may be considered known. Thus, Eq. (5) determines the variation of along a field line. The resultant $\Phi$ will be single valued, as is necessary, by virtue of Eq. (3). The variation of from field line to field line is free and connected with a corresponding freedom in the velocity $\nabla$. The velocity perpendicular to $B$ is determined as the last step by crossing Eq. (1) with $B$ to obtain

$$
v_{1}=\frac{1}{B^{2}} B \times\left(\eta j+\frac{\partial A}{\partial t}+\nabla_{\Phi}\right)
$$

The velocity parallel to $B$ is not determined, as is clear from Eq. (1).

\section{I1. EQUILIBRIUM RELATIONS}

The equilibrium relations for a plasma with magnetic surfaces as well as general nomenclature used in this report are briefly developed in this section. The basic equilibrium equations are given by Eqs. (2). We assume the magnetic field lines form nested toroidal surfaces that are labeled by a surface-dependent quantity $S$ so that B. VS $=0$. Equation (2a) implies that $B \cdot \nabla_{p}=0$, and if a field line traces out the entire magnetic surface, pressure must be constant over the surface. Thus, $p=p(S)$ and

$$
\nabla_{\mathbf{p}}=\mathbf{p}^{\prime} \nabla_{\mathbf{S}}
$$

where" denotes the derivative d/dS. From Eq. (2a), j. $\nabla S=0$ and $j$ and $B$ may be represented as follows:

$$
\begin{aligned}
& j=\nabla_{S} \times \nabla_{\mu} \\
& B=\nabla_{S \times} \nabla_{\nu}
\end{aligned}
$$

where, 23 


$$
\begin{aligned}
& \mu=\int_{S} \frac{j \times \nabla S}{|\nabla S|^{2}} \cdot d r \\
& \nu=\int_{S} \frac{B \times \nabla S}{|\nabla S|^{2}} \cdot d r
\end{aligned}
$$

Another surface function of use is defined as follows:

$$
\zeta=\int_{S} B \cdot \mathrm{d} r
$$

Note that the quantities defined by Eqs. (9a), (9b), and(10) are independent of the path of integration but multiple valued. The periodicity of these functions is given by

$$
\begin{aligned}
& \mu=\mathrm{I} u+\mathrm{J} v+\alpha \\
& \nu=\hat{\psi}^{\prime} \mathrm{u}+\mathrm{X}^{\prime} v+\hat{\boldsymbol{p}} \\
& \zeta=\mathrm{I} \mathrm{u}+\mathrm{J} v+\gamma
\end{aligned}
$$

where $u$ and $v$ are multiple-valued angle coordinates that increase by 1 in each circuit of the toroidal magnetic surface the short way around and the long way around, respectively, and $\alpha, \beta$, and $\gamma$ are periodic functions of $u$ and $v$. The quantities $I$ and $\psi$ are the toroidal current and $f$ lux through the minor cross section of the torus, while $J$ and $x$ are the foloidal current and $f$ lux through the mejer cross section of the torus. Note that this definition of $J$ and $x$ is nonstandard and differs, for example, from that of Ref. 23. From Eqs. (8b) and $(10)$,

$$
\nabla_{S} \times \nabla_{\nu} \cdot \nabla_{\zeta}=\mathrm{B}^{2}>0
$$


and $S, \nu$, and $\zeta$ thus can be used to define a coordinate system.

The equilibrium relation Eq. (2a) can be written

$$
\mathbf{p} \cdot \nabla S=-B \cdot \nabla_{\mu} \nabla S
$$

or

$$
\left.\mathrm{B}^{2} \frac{\partial \mu}{\partial \zeta}\right|_{\mathrm{S}, \nu}=-\mathrm{p}^{\circ}
$$

The general solution 24 of this equation with the required periodicity is

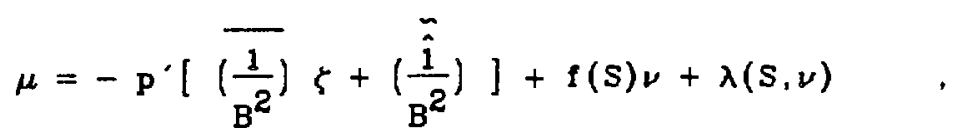

where $\lambda$ is periodic in $\nu$. The following notation has been used:

$$
\begin{aligned}
& \bar{F}=\frac{\int_{0}^{\infty} F d \zeta}{\int_{0}^{\infty} d \zeta} \\
& \tilde{F}=F-\bar{F} \\
& \hat{F}=\int_{0}^{\zeta} F d \zeta
\end{aligned}
$$

To have $\mathrm{j}=\nabla_{S} \times \nabla_{\mu}$ single valued, it is further necessary that 


$$
p \cdot \frac{\partial}{\partial \nu}\left[\overline{\left(\frac{1}{B^{2}}\right)}\right]=0
$$

which translates to the well-known condition that on a rational surface either

$$
\text { f } \frac{\mathrm{d} \ell}{B}=\text { constant }
$$

from field line to field line or

$$
\mathbf{p}^{\prime}=\mathbf{0}
$$

Evaluating the periods of $\mu$ from Eq. (15) and comparing with Eq. (11a) leads to the following equaities:

$$
\begin{aligned}
& -p^{\prime}\left(\overline{\frac{1}{B^{2}}}\right) I+1 \psi^{\prime}=I^{\prime} \\
& -p^{\prime}\left(\overline{\frac{1}{B^{2}}}\right) \mathrm{J}+1 x^{\prime}=J^{\prime}
\end{aligned}
$$

Furthermore,

$$
\left(\frac{1}{B^{2}}\right)=\frac{\int \frac{d l}{B}}{\int B \cdot d l}
$$

The integrand in the numerator may be considered a volume element of a tube of $f l u x$ by use of the relation 


$$
d V=d A d \ell=d \varphi \frac{d \ell}{B}
$$

where $d \varphi$ is the $f l u x$ in the $f l u x$ tube with transverse area dA. This gives

$$
\int \frac{d \ell}{B}=N_{v} \frac{d V}{d \psi}=-N_{u} \frac{d V}{d x}
$$

where $N_{u}$ and $N_{v}$ are the number of circuits of the path of integration (a field line) the short way around the torus and the long way around the torus, respectively. The origin of the minus sign in Eq. (20) is the fact that if $x$. and $\psi$ increase as one goes away from the magnetic axis (in $V$ ), then the field line rotates in a negative (left-hand) sense around the magnetic axis.

The denominator in Eq. (18) may be written as

$$
\int B \cdot d \ell=N_{v}(\not L+J)
$$

where $\ell \equiv N_{u} / N_{v}$. Thus, Eq. (18) becomes

$$
\overline{\left(\frac{1}{B^{2}}\right)}=\frac{\frac{d V}{d \psi}}{A I+J}
$$

Equations (17a) and $(17 b)$ can he solved for $p^{\prime}$ and $f$, yielding

$$
\begin{aligned}
& p^{\prime}=-\frac{\not I^{\prime}+J^{\prime}}{d V / d \psi} \\
& I=\frac{J \frac{d I}{d \psi}-I \frac{d J}{d \psi}}{\not I+J}
\end{aligned}
$$


where we have used the relation $l=-\mathrm{d} \chi / \mathrm{d} \psi$.

The current parallel to the magnetic field is conveniently specified by the quantity

$$
\sigma \equiv \frac{j \cdot B}{B^{2}}=\frac{\nabla_{S \times \nabla} \cdot \nabla_{\zeta}}{B^{2}}=\left.\frac{\partial \mu}{\partial \nu}\right|_{S, \zeta}
$$

From Eq. (15),

$$
\sigma=-p^{\prime} \frac{\partial}{\partial \nu}\left(\frac{\hat{\hat{i}}}{B^{2}}\right)+f+\frac{\partial \lambda}{\partial \nu}
$$

and

$$
\bar{\sigma}=f+\frac{\partial \lambda}{\partial \nu}
$$

where the general relation $\overline{\bar{F}}=0$ is used. As $B \cdot \bar{\nabla} \bar{\sigma}=B^{2} \partial \bar{\sigma} / \partial \zeta=0$, one can argue that $\bar{\sigma}$ is constant on a magnetic surface in complete analogy to the argument that $p$ is constant on a magnetic surface (specific properties of rational surfaces are overlooked in either case). If $\bar{\sigma}=\bar{\sigma}(\mathrm{s})$, the terms $\lambda(S, \nu)$ in Eq. (15) must be zero, and we have simply

$$
\bar{\sigma}=\mathbf{f}
$$

\section{EVOLUTION OF A MAGNETIC FIELD WITH MAGNETIC SURFACES}

Consider a particular rational magnetic surface, a toroidal surface upon which field lines close on themselves after $N_{u}$ circuits the short way around 
and $N_{v}$ circuits the long way around. Integrating $\mathrm{Eq}$. (1) along a field line gives the result

$$
\mathrm{N}_{\mathrm{v}} \frac{\partial \chi}{\partial t}+\mathrm{N}_{\mathrm{u}} \frac{\partial \psi}{\partial t}=-\eta \oint j \cdot \mathrm{d} \ell
$$

where it is assumed that $\eta$ is constant along a field line. The left-hand side of Eq. (28) can be written as

$$
\mathrm{N}_{\mathrm{v}} \frac{\partial}{\partial \mathrm{t}}(\chi+\ell \psi)_{\ell}=\mathrm{N}_{\mathrm{v}}\left[\left.\frac{\partial \chi}{\partial \mathrm{t}}\right|_{\mathrm{S}}+\left.\ell \frac{\partial \psi}{\partial \mathrm{t}}\right|_{\mathrm{S}}+\left.\left(\frac{\partial \chi}{\partial \mathrm{S}}+\not \frac{\partial \psi}{\partial \mathrm{s}}\right) \frac{\partial \mathrm{S}}{\partial \mathrm{t}}\right|_{\ell}\right]
$$

or

$$
\mathrm{N}_{\mathrm{v}} \frac{\partial}{\partial \mathrm{t}}(x+\ell \psi)_{\ell}=\mathrm{N}_{\mathrm{v}}\left(\left.\frac{\partial x}{\partial \mathrm{t}}\right|_{\mathrm{S}}+\left.\not \frac{\partial \psi}{\partial \mathrm{t}}\right|_{\mathrm{S}}\right)
$$

with $\ell \equiv \mathrm{N}_{\mathrm{u}} / \mathrm{N}_{\mathbf{v}}=-\mathrm{d} \chi / \mathrm{d} \psi$, which follows from Eq. (20). Letting $\mathrm{s}=\psi$, we obtain

$$
\left.\frac{\partial x}{\partial t}\right|_{\psi}=-\frac{n t i \cdot d \ell}{N_{v}}
$$

Taking the derivative with respect to $\psi$, we obtain

$$
\left.\frac{\partial \ell}{\partial t}\right|_{\psi}=\frac{\partial}{\partial \psi}\left(\frac{\eta \phi j \cdot d \ell}{\mathrm{N}_{v}}\right)
$$

The right-hand side of Eq. (31) involves the integral 


$$
\phi \cdot d \ell=\emptyset \sigma d \zeta=\bar{\sigma} \Delta \zeta \quad \text {, }
$$

where $\Delta \zeta=\mathbb{I N}_{\mathrm{u}}+\mathrm{JN}_{\mathrm{v}}=\mathrm{N}_{\mathrm{v}}(\not I+J)$.

From Eq. (27) and (23b)

$$
\bar{\sigma}=f=\frac{J \frac{\partial I}{\partial \psi}-I \frac{\partial J}{\partial \psi}}{\not I+J}
$$

which leads to the result ${ }^{18}$

$$
\begin{aligned}
\left.\frac{\partial \not \partial}{\partial t}\right|_{\psi} & =\frac{\partial}{\partial \psi}\left[\eta\left(\mathrm{J} \frac{\partial \mathrm{I}}{\partial \psi}-\mathrm{I} \frac{\partial \mathrm{J}}{\partial \psi}\right)\right] \\
& =-\frac{\partial}{\partial \psi}\left[\eta \mathrm{I}^{2} \frac{\partial}{\partial \psi}\left(\frac{\mathrm{J}}{\mathrm{I}}\right)\right] \\
& =\frac{\partial}{\partial \psi}\left[\eta \mathrm{J}^{2} \frac{\partial}{\partial \psi}\left(\frac{\mathrm{I}}{\mathrm{J}}\right)\right]
\end{aligned}
$$

The quantities $\mathcal{L}, \psi, I, J$, and $\eta$ appearing in Eq. (32) are, respectively, rotational transform, toroidal flux, toroidal current, poloidal current, and resistivity. By a straightforward change of dependent and independent variables $\ell \rightarrow q \equiv 1 / \ell, \psi \rightarrow \chi$. Eq. (32) can also be written as ${ }^{18}$

$$
\left.\frac{\partial \mathrm{g}}{\partial \mathrm{t}}\right|_{\chi}=\frac{\partial}{\partial x}\left[\eta\left(\mathrm{J} \frac{\partial \mathrm{I}}{\partial x}-1 \frac{\partial \mathrm{J}}{\partial x}\right)\right]
$$

It is known $23,25,26$ that magnetostatic equilibria are specified by the two functions $f(\psi)=-d \chi / d \psi$ and $d w / d \psi$, where 


$$
M(\psi)=\int \mathrm{p}^{1 / \gamma} \mathrm{d} \tau
$$

with the integration volume bounded by the magnetic surface $\psi$ (the quantity $M$ is related to the total number of particles contained within surface $\psi$ as well as their entropy). Thus, Eq. (32) and a corresponding equation for the rate of change of $\mathrm{dM} / \mathrm{d} \psi$, which is not discussed here, describe the evolution of plasma equilibria with given flux-surface topology. The process of change of flux-surface topology, or magnetic island formation, is not described by Eq. (32); however, the subsequent growth or decay of islands, once introduced, is. An inconsistency of the equilibrium evolution model occurs for very small islands in that the resistive diffusion time, which scales like $w^{2} / \eta$, where w is the island width, can become short compared with the time for an inertial disturbance to propagate accross the island, invalidating the neglect of acceleration terms in the equation of motion. Roughly speaking, as long as the islands are sufficiently large that the resistive diffusion time exceeds the inertial time (Alfven or magnetosonic time), the equilibrium description of the plasma remains valid. ${ }^{2}$ ' In the low-density limit, the critical island size vanishes, and the equilibrium evolution model offers a complete description of plasma behavior.

Such a complete description involves tests for stability toward islation of each (low-order) rational surface as time proceeds. In a cylindrical plasma. 28 stability toward islation is determined in a straightforward way by examining neighboring plasma equilibria, and this fairly clearly remains true in the general case. Stability is actually determined by boundary conditions rather than by properties of Eq. (32) itself. In the complete description of plasma behavior, the islation stability test must be carried out and if the plasma is unstable, a small island introduced and the evolution continued.

Examples of Eq. (32) in simple geometries are of interest. For a cylinarical plasma of length $2 \pi R$, described in cylindrical coordinates $r, v$, and $z$, 


$$
\begin{aligned}
& \ell=\frac{\mathrm{RB}_{\mathfrak{v}}}{\mathrm{rB}_{\mathbf{z}}} \\
& \mathrm{I}=2 \pi \mathrm{r} \mathrm{B}_{\boldsymbol{v}} \\
& \mathrm{J}=2 \pi \mathrm{RB}_{\mathbf{z}}
\end{aligned}
$$

and Eq. (32c) becomes

$$
\frac{\partial}{\partial t}\left(\frac{\mathrm{B}_{\vartheta}}{\mathrm{rB}_{\mathrm{z}}}\right)_{\psi}=\frac{\partial}{\partial \psi}\left[\eta \mathrm{B}_{\mathrm{z}}^{2} \frac{\partial}{\partial \psi}\left(\frac{\mathrm{rB}_{\psi}}{\mathrm{B}_{\mathrm{z}}}\right)\right]
$$

where $d \psi=\mathrm{rB}_{z} \mathrm{dr}$.

In slab geometry $(r, v, z \rightarrow x, y, z)$, the equilibrium fields are of the form

$$
\begin{aligned}
& B_{y}=B \sin \varphi \\
& B_{z}=B \cos \varphi
\end{aligned}
$$

with $\mathrm{B}=\left(\mathrm{B}_{0}^{2}-2 \mathrm{p}\right)^{1 / 2}$, where $\mathrm{B}_{0}$ is the field outside the plasma region, and Eq. (34) reduces to

$$
\frac{\partial}{\partial \mathrm{t}}(\tan \varphi)_{\psi}=\frac{\partial}{\partial \psi}\left(\eta \mathrm{B}^{2} \frac{\partial \varphi}{\partial \psi}\right)
$$

or

$$
\left.\frac{\partial \varphi}{\partial t}\right|_{\psi}=(\cos \varphi)^{2} \frac{\partial}{\partial \psi}\left(\eta B^{2} \frac{\partial \varphi}{\partial \psi}\right)
$$


so clearly $\varphi(\psi, t)$ has a simple diffusive character.

Equation (36) is equivalent to a Eulerian form used by other authors. The $t$ ime derivative in $\mathrm{Eq}$. (36) is given by

$$
\left.\frac{\partial \varphi}{\partial \mathrm{t}}\right|_{\psi}=\left.\frac{\partial \varphi}{\partial \mathrm{t}}\right|_{\mathbf{x}}+\left.\frac{\partial \varphi}{\partial \mathrm{x}} \frac{\partial \mathrm{x}}{\partial \mathrm{t}}\right|_{\psi}=\left.\frac{\partial \varphi}{\partial \mathrm{t}}\right|_{\mathrm{x}}-\frac{\partial \varphi}{\partial \mathrm{x}} \frac{\frac{\partial \psi}{\partial \mathrm{t}}}{\frac{\partial \psi}{\partial \mathrm{x}}}
$$

leading to the following Eulerian equation in the pressureless case:

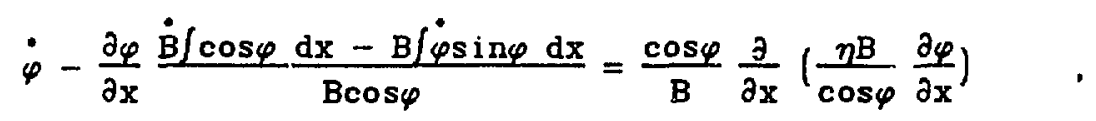

where the $\operatorname{dot}\left({ }^{*}\right)$ denotes the derivative with respect to time. Equation is of the form

$$
f+\frac{\varphi^{\prime}}{\cos \varphi} \int_{0}^{x} f \sin \psi d x=g
$$

where $f=\partial \varphi / \partial t$ and the prime (') denotes $\partial / \partial x$. The above integral equation is equivalent to the differential equation

$$
\left(\frac{f}{\varphi^{\prime}}\right)^{\prime}=\left(\frac{g \cos \varphi}{\varphi^{\prime}}\right)^{\prime} \frac{1}{\cos \varphi}
$$

Thus, we have the inversion

$$
f=\varphi^{\prime} \int_{0}^{x} \frac{1}{\cos \varphi}\left(\frac{\rho \cos \varphi}{\varphi^{\prime}}\right)^{\prime} \mathrm{dx}
$$


Judiciously integraing the right-hand side above by parts and using the fact that $\varphi$ is odd about $x=0$, we obtain the desired form

$$
\frac{\partial \varphi}{\partial t}-\frac{\partial \varphi}{\partial x}\left[\frac{\dot{B}}{B} \mathbf{x}+\int_{0}^{x} \eta\left(\frac{\partial \varphi}{\partial \mathbf{x}}\right)^{2} \mathrm{~d} \mathbf{x}\right]=\frac{\partial}{\partial \mathbf{x}}\left(\eta \frac{\partial \varphi}{\partial \mathbf{x}}\right)
$$

which is an equation given by Low. 5

Low 5 has pointed out that the slab model of resistive diffusion can exhibit a curious behavior in the high-current regime. In certain cases, profiles develop infinite gradients in a finite time rather than relaxing to a stationary state. This behavior is probably not physically relevant because of the neglect of islation. In fact, high-current profiles are very unstable toward islation and would probably evolve into a fully stochastic state. leading to a different description of the evolution process.

IV. EVOLUTION OF AN ERGODIC MAGNETIC FIELD

We consider a region containing a stochastic magnetic field bounded by a magnetic surface. Equation (1), when integrated along a field line, is

$$
-\phi \frac{\partial A}{\partial t} \cdot B \frac{d \ell}{B}=\eta \oint j \cdot B \frac{d \ell}{B}
$$

We assume that the field line ergodically fills the entire stochastic volume. Using Eq. (19), we may interpret $d \ell / B$ as a volume element, leading to the relation

$$
-\int \frac{\partial A}{\partial t} \cdot B d \tau=\eta \int j \cdot B d \tau \quad
$$

where $d \tau$ is an element of volume. 
Equation (40) constitutes the constraint imposed by Ohm's law in the case of an ergodic field. This single relation is sufficient because ergodicity severely constrains the form of the plasma equilibrium, implying (1) that $p$ is spaicially constant throughout the ergodic region from the relation $B \cdot \nabla_{p}=0$ and the assumption of ergodicity, and (2) that $\sigma$ is also spatially constant from the relation $j=\sigma B$ and $i$ ts divergence, which gives $B \cdot \nabla_{\sigma}=\nabla \cdot j=0$. The magnetic field thus satisfies (approximately, in some limiting sense)

$$
\nabla \times B=\sigma B \quad,
$$

with $\sigma$ spatially constant, and such a field is completely determined by the value of $\sigma$ and a normalization factor. These two quantities are specified by two equations, one of wh:ch is Eq. (40). The second equation might be an equation for the toroidal flux if this is imagined to be specified externally. Equation (40) can be cast into a form more closely related to the work of J. B. Taylor 29,30 by defining

$$
\left.K(S)=\frac{1}{2} \int A \cdot B d \tau-\frac{1}{2}: X \psi\right],
$$

where the integral is taken over the ergodic region, $\psi$ is the toroidal flux within the ergodic region, $x$ is the poloidal flux at the bounding magnetic surface $S=C$, and the square brackets denote the difference in the enclosed quantity between the outer and inner surfaces. The reason for this definition of $K,{ }^{31.32}$ slightly different from that originally proposed by Taylor, is that it depends only on the magnetic field within the ergodic volume and not on the volt.second history of the plasma (i.e., the gauge of A). We then find that (assuming $S$ monotonically increases with volume)

$$
\frac{d K}{d t}=\frac{1}{2} \int\left(\frac{\partial A}{\partial t} \cdot B+A \cdot \frac{\partial B}{\partial t}\right) d \tau+\frac{1}{2}\left[\frac{d C}{d t} \int \frac{A \cdot B}{|\nabla S|} d \sigma-\left(\frac{d x}{d t} \psi+\chi \frac{d \psi}{d t}\right)\right]
$$




$$
=\int \frac{\partial A}{i t} \cdot B d \tau-\frac{1}{2}\left(\int A \times V \cdot d \sigma\right)-\frac{1}{2}\left[\frac{d \gamma}{d t} \psi+x \frac{d \psi}{d t}\right]
$$

where do is an element of the bounding magnetic surface $S=C$ and

$$
v \equiv \frac{\partial A}{\partial t}+\frac{B \times \nabla S}{|\nabla S|^{2}} \frac{d C}{d t}
$$

In the surface integral, $A$ can be replaced by $\nabla \lambda$, where $\lambda$ is the multiple-valued function

$$
\lambda=\int_{S} \mathbf{A} \cdot \mathbf{d} \mathbf{r}
$$

The fact that $\nabla \times A \cdot d \sigma=0$ means the line integral is independent of path. However, because of multiple values, the surface of integration (the magnetic surface bounding the ergodic region) must be cut in the toroidal and poloidal directions. In terms of $\lambda$, the surface integral can be written as

$$
\begin{aligned}
\int A \times v \cdot d \sigma & =\int \nabla \times(\lambda v) \cdot d \sigma \\
& =[\lambda]_{u} \oint v \cdot d \ell_{v}-[\lambda]_{v} \oint v \cdot d \ell_{u},
\end{aligned}
$$

where $\nabla \times V \cdot d \sigma=0$, which follows from $B \cdot d \sigma=0$ and $\nabla \cdot B=0$, where $B$ is written as $\nabla S \times w$ with $w=B \times \nabla S /|\nabla S|^{2}$. Using

$$
\begin{aligned}
& \oint v \cdot d l_{v}=\frac{d x}{d t} \\
& 6 v \cdot d l_{u}=\frac{d y}{d t},
\end{aligned}
$$


we obtain

$$
\begin{aligned}
\frac{d K}{d t} & =\int \frac{\partial A}{\partial t} \cdot B d \tau-\left[\psi \frac{d \chi}{d t}\right] \\
& =-\int \eta j \cdot B d r-\left[\psi \frac{d \chi}{d t}\right]
\end{aligned}
$$

The quantity $K$ can be taken as one of the two parameters specifying the ergodic field state, with $K$ evolving according to Eq. (44). The significance of Eq. (44) for the problem of resistive evolution is the main new result of this report.

For a stochastic magnetic field not bounded by a magnetic surface, the ergodic assumption is not valid because individual field ilnes begin and end on external boundaries. Such a configuration belongs to the class of open rather than ciosed magnetic topologies and would seem to be fundamentally different regarding the effect of resistivity.

A simple example of resistive evolution of an ergodic magnetic fieid is such a field contained within a perfectly conducting cylinder. A solution of $\nabla \times B=\sigma B$ satisfying the wall boundary condition $\left(B_{r}=0\right)$ is

$$
\begin{aligned}
& \mathrm{B}_{\vartheta}=\mathrm{B}_{0} \mathrm{~J}_{1}(\sigma \mathrm{r}) \\
& \mathrm{B}_{\mathrm{z}}=\mathrm{B}_{0} \mathrm{~J}_{0}(\sigma \mathrm{r}),
\end{aligned}
$$

with $J_{0}$ and $J_{1}$ the Bessel functions. We are free to choose $A=B / \sigma$, which gives

$$
\begin{aligned}
& K=B_{0}^{2} \frac{2 \pi^{2} R}{\sigma}\left[\int_{0}^{a} r\left(J_{0}^{2}+J_{1}^{2}\right) d r-J_{0}(\sigma a) \int_{0}^{a} r J_{0} d r\right] \\
& \psi=2 \pi B_{0} \int J_{0} r d r
\end{aligned}
$$


and

$$
\frac{\mathrm{K}}{\psi^{2}}=\frac{\mathrm{R}}{2 \mathrm{a}}\left(\mathrm{x}\left[1+\frac{\mathrm{J}_{0}^{2}(\mathrm{x})}{\mathrm{J}_{1}^{2}(\mathrm{x})}\right]-\frac{2 \mathrm{~J}_{0}(\mathrm{x})}{\mathrm{J}_{1}(\mathrm{x})}\right)
$$

where $x=\sigma a$ with a the wall radius. Equation $(44)$, with $d x / d t=0$ at the wall, implies that

$$
\frac{\mathrm{d}}{\mathrm{dt}}\left(\frac{\mathrm{K}}{\psi^{2}}\right)=-\frac{\eta \sigma}{\psi^{2}} / \mathrm{B}^{2} \mathrm{~d} \tau
$$

Therefore, $K / \psi^{2}$ decays with time. Since $K / \psi^{2}$ given by $\mathrm{Eq}$. (46) is a monotonically increasing function of $\sigma, \sigma$ also decays with time. The equation for toroidal flux gives $\mathrm{dB}_{0} / \mathrm{dt}$ in terms of $\mathrm{d} \sigma / \mathrm{d} t$ and completes the determination of the $t$ ime dependence of the magnetic field.

For this solution, $(\partial A / \partial t+\eta j) \cdot B$ does not vanish locally. If it did, one would obtain the evolution of a nonergodic cylindrical field. From Eq. (5), the potential therefore has a complicated structure depending on the detailed stochastic structure of the magnetic field. Likewise, $v_{1}$ has a complicated structur, containing singularities where a finite potential difference occurs between field lines that are arbitrarily close at some point. These singularities would probably be eliminated by other physical effects in a more correct treatment. In addition to the problem with velocity singularities, the detailed stochastic structure of the magnetic field or its time evolution have not been determined. Indeed, an important unresolved question is exactly how the solutions of $\nabla \times B=\sigma B$ with magnetic surfaces are to be interpreted as limiting cases of stochastic fields.

We can obtain the evolution equation for a plasma with magnetic surfaces. Eq. (32), from the equation for K, Eq. (44). Thus, the $K$ equation may be taken as the general law of resistive evolution of a plasma. First we note 
the following relations concerning an infinitesinal plasma volume bounded by magnetic surfaces $S=C$ and $S=C+d C$.

$$
\begin{aligned}
& d\left(\int A \cdot B d \tau\right)=\chi d \psi-\psi d \chi \\
& d\left(\int B \cdot j d \tau\right)=J d I-I d J
\end{aligned}
$$

These relations can be proved using a substitution of the type given by Eq. (43) and arguments similar to those used in obtaining Eq. (44).23 If Eq. (48) is used for a plasma with magnetic surfaces, $K$ is given by

$$
\mathrm{K}=-\int \psi \mathrm{d} x=\int \psi \not h \mathrm{~d} \psi
$$

This equation had been proposed ${ }^{33}$ as a definition of $K$, although clearly the form given by Eq. (42) is preferrable because of its greater generality. Equation (44) for the time derivative of $\mathrm{K}$ leads to

$$
\left.\frac{\partial K}{\partial t}\right|_{\psi}+\frac{\partial K}{\partial \psi} \frac{d \psi}{d t}=-\psi\left(\left.\frac{\partial x}{\partial t}\right|_{\psi}+\frac{\partial \chi}{\partial \psi} \frac{d \psi}{d t}\right)-\int \eta j \cdot B d \tau
$$

where the lower boundary of the integration is taken at $\psi=0$. However, from Eq. (49),

$$
\frac{\partial K}{\partial \psi}=\psi \ell=-\psi \frac{\partial \chi}{\partial \psi}
$$

so that

$$
\left.\frac{\partial K}{\partial t}\right|_{\psi}=-\left.\psi \frac{\partial x}{\partial t}\right|_{\psi}-\int \eta j \cdot B d r
$$


Taking the derivative with respect to $\psi$, we obtain

$$
\frac{\partial^{2} \mathrm{~K}}{\partial \mathrm{t} \partial \psi}=\left.\psi \frac{\partial \ell}{\partial \mathrm{t}}\right|_{\psi}=-\left.\frac{\partial \chi}{\partial \mathrm{t}}\right|_{\psi}+\left.\psi \frac{\partial \ell}{\partial \mathrm{t}}\right|_{\psi}-\frac{\partial}{\partial \psi} \int \eta \mathrm{j} \cdot \mathrm{B} \mathrm{d} \tau
$$

implying

$$
\left.\frac{\partial \chi}{\partial t}\right|_{\psi}=-\eta \frac{\partial}{\partial \psi} \int \mathbf{j} \cdot \mathrm{B} \mathrm{d} \tau=\eta\left(\mathrm{I} \frac{\partial \mathrm{J}}{\partial \psi}-\mathrm{J} \frac{\partial \mathrm{I}}{\partial \psi}\right)
$$

Taking another $\psi$ derivative leads back to Eq. (32) or the alternate form

$$
\left.\frac{\partial \ell}{\partial t}\right|_{\psi}=\frac{\partial}{\partial \psi}\left[\eta \frac{\partial}{\partial \psi} \int \mathrm{j} \cdot \mathrm{Bd} \tau\right]
$$

\section{OHMIC STATES}

In this section, the properties of steady states of resistive evolution are exemined. Consider a plasma bounded by a toroidal surface on which there are prescribed toroidal and poloidal voltages $v_{v}$ and $v_{u}$. In a steady state, the voltages are constant in $t$ ime. As $v_{u}=-\partial \psi / \partial t, v_{u}$ must be zero. Similarly, $V \equiv v_{v}=-\partial \chi / \partial t$, although $V$ is not necessarily taken to be zero. However, the poloidal flux within the plasma is assumed constant in time. The toroidal voltage $V$ is then the same throughout the plasma.

For a plasma with magnetic surfaces, Eq. (30) gives

$$
v=\frac{n+f_{v} \cdot d \ell}{\mathrm{N}_{v}}
$$




$$
v=\eta\left(\mathbf{J} \frac{\partial \mathrm{I}}{\partial \psi}-1 \frac{\partial \mathrm{J}}{\partial \psi}\right)
$$

or

$$
V=\eta \frac{\partial}{\partial \psi}\left(\int j \cdot B d \tau\right)
$$

In a true steady state, $\mathbf{V}=\mathbf{0}$. Such configurations are stellarators, or, in more general terms, plasma without externally driven currents. Ohmic states of a stellarator satisfy

$$
\eta \mathrm{J}^{2} \frac{\partial}{\partial \psi}\left(\frac{\mathrm{I}}{\mathrm{J}}\right)=0
$$

which implies that $I=\alpha J$ with $\alpha$ constant. Since $I$ is zero on the magnetic axis, but $J$ is not ( $J_{0}=\int B \cdot d l_{0}$ includes the currents in the external coils). a must be zero. Thus, we obiain the condition that a stellarator has zero net current on each flux s'rface in a steady state. 23 if a steady state stellarator contains an argodic magnetic field region, then $j=\sigma B$ within that region, and Eq. (44) implies that $\sigma=0$. Since pressure is uniform in the ergodic region, this region is completely current free and is effectively a vacuum field region.

The Ohmic condition for a cylindrical plasma with magnetic surfaces can be written as

$$
\frac{V}{2 \pi R}=\frac{\eta \sigma \mathrm{B}^{2}}{\mathrm{~B}_{\mathrm{z}}}
$$

from Eq. (53), while for ergodic fields, only the integral relation 


$$
\frac{\mathrm{V}}{2 \pi R} \int \mathrm{B}_{\mathrm{z}} \mathrm{rdr}=\eta \mathrm{B}^{2} \mathrm{rdr}
$$

applies. Ergodic Ohmic states in a cylinder are given by Eq. (45), with the above relation determining the necessary driving voltage.

In the case of magnetic surfaces, Eq. (52) leads to the following alternate form:

$$
\frac{v}{2 \pi R}=-\eta \frac{r B_{\vartheta}^{2}}{B_{z}} \frac{\partial}{\partial r}\left(\frac{B_{z}}{r B_{\vartheta}}\right)
$$

Within the context of the model, cylindrical Ohmic states cannot have a reversal of sign of $B_{z}$. If $B_{z}$ did reverse at some point, immediately outside this point, $B_{z}$ and $\partial\left[B_{z} /\left(\mathrm{rB}_{\vartheta}\right)\right] / \partial r$ would be negative, leading to a contradiction in the signs of the left-and right-hand sides of Eq. (56). Ergodic Ohmic states in a cylinder are given by Eq. (45) and can have sustained $B_{z}$ reversal. Another interpretation of sustainment of reversal is given in Ref. 34.

In the slab model, Ohmic states satisfy

$$
E=\eta B^{2} \frac{\partial \varphi}{\partial \psi}
$$

where $E$ is the driving electric field, $B_{y}=B \sin \varphi$, and $B_{z}=B \cos \varphi$. In the pressureless case with uniform $\eta$, Eq. (57) implies

$$
\varphi=\frac{\mathrm{E}}{\eta \mathrm{B}^{2}} \psi
$$


where we use the fact that $\varphi$ and $\psi$ vanish at $x=0$. Inserting the definition of $\psi$,

$$
\varphi=\frac{E}{\eta B} \int_{0}^{x} \cos \varphi\left(x_{1}\right) d x_{1}
$$

and

$$
\frac{\mathrm{d} \varphi}{\cos \varphi}=\frac{E}{\eta B} \mathrm{~d} \mathbf{x}
$$

The solution of this equation that has $\varphi=0$ at $x=0$ is given by

$$
\varphi=\tan ^{-1}\left[\sinh \left(\frac{E x}{\eta B}\right)\right]
$$

Note that as $E+\infty, \varphi+\pi / 2$ so that $B_{z}+0$ but never reverses sign.

The perpendicular flow velocity for the cylindrical case with magnetic surfaces is determined from the general relation Eq. (6), with $\Phi=0$ by symmetry in this case, giving

$$
\nabla_{\mathrm{r}}=-\frac{\mathrm{v}}{2 \pi \mathrm{R}} \frac{\mathrm{B}_{\vartheta}}{\mathrm{B}^{2}}-\frac{\eta}{\mathrm{B}^{2}} \frac{\mathrm{dp}}{\mathrm{dr}}
$$

In the pressureless case, the flow is inward. Equation (54) applied on axis ident ifies $\mathrm{V}$ as

$$
\frac{V}{2 \pi R}=\eta(0) \sigma(0) B(0)
$$


which when substituted in Eq. (59) yields a condition determining steady-state pressure profiles with no inward pinching. The steady-state pressures are found to be quite large. Stability investigations 28 indicate that in the high-current regime, such cylindrical Ohmic states, even with no pressure, are extremely unstable toward islation. This suggests that sustained high-current cylindrical discharges would be in a stochastic state.

\section{vi. CONCLUSIONS}

The resistive evolution of magnetic fields with closed field lines is specified in a simple and natural way by the equation for the rate of change of magnetic flux linking those closed lines. For the case of magnetic surfaces, closed field lines exist on each rational surface, leading to the equation describing the time rate of change of rotational transform on each such surface. For an ergodic magnetic field region, however, a single magnetic field line is imagined to trace out the entire region and $f$ lux linkages are defined by the quantity $K$ and associated with the entire ergodic volume. A geometric interpretation of $K$ as the degree of "knottedness" of tangled filaments has been given by Moffatt. ${ }^{35}$ The equation for the time rate of change of $K$ can in fact be taken as the general law of resistive evolution as it implies the previous result for megnetic surfaces as a special case.

The energy priciple of Kruskal and Kulsrud 23 can be restated to include ergodic regions as follows: steble magnetohydrodynamic equilibria are minima of the potential energy, with the constraint that the function $\mathrm{dK} / \mathrm{d} \psi^{2}=\not(\psi) / 2$ is given on magnetic surfaces, while the quantity $K / \psi^{2}$ is given for ergodic field regions (the pressure is imagined to be specified in either case). The process by which a plasma reaches the minimum potential energy state consistent with the particular flux constraints of the problem is the dissipation of kinetic energy through viscosity or other means. This "relaxation" process takes place on the inertial time scale. Relaxation as defined by J. B. Tayior takes place by an unspecified process. Taylor relaxation is the tendency of a plasma, whatever its initial flux constraints, to approach a unique final minimum energy state. For a plasma in a perfectly conducting chamber, the minimum potenial energy state associated with a given total $\mathrm{K}$ in the chamber is the Taylor state with $\sigma$ spatially uniform. Thus, 
insofar as the resistive dissipation of $\mathrm{K}$ is negligible. 36,28 the plasma will be forced by resistive dissipation of potential energy into the Taylor state.

clearly, one interpretation of Taylor states is that they are configurations with ergodic field lines, as has already been suggested. $37-39$ This report provides a more complete conceptual framework for dealing with such configurations.

Although many questions remain, the resistive evolution model would seem in principle to offer a complete description of long-time-scale plasma behavior. The process of island formation or change of magnetic topology is not described by the evolution equations, but the growth or decay of islands or ergodic regions, once introduced, is. Thus, a complete description of plasma behavior would involve tests for stability toward change of magnetic topology by introducing (conceptually, if not actually, in computations) such changes and observing whether the effects grow or decay with time. 


\section{REFERENCES}

1. H. Grad and J. Hogan, Phys. Rev. Lett. 24, 1337 (1970).

2. J. Andreoletti, J. Plasme Phys. 3, 313 (1971).

3. J. Nuhrenburg, Nucl. Fusion 12, 157 (1972).

4. B. C. Low, Astrophys. J. 181, 209 (1973).

5. B. C. Low, J. Plasma Phys. 24, 181 (1980).

6. B. C. Low, Phys. Fluids 25, 492 (1981).

7. A. Kadish and J. W. A. Zwart. Phys. Fluids. 16. 2190 (1973).

8. A. Kadish and J. W. A. Zwart, J. Plasma Phys. 16, 43 (1974).

9. A. Kadish, J. Plasma Phys. 17, 555 (1975).

10. H. Grad, P. N. Hu, and D. C. Stevens, Proc. Nat. Acad. Sci. USA 72, 3789 (1975).

11. H. Grad, "Survey of $11 / 2$ D Transport Codes," Courant Institute of Mathematical Sciences report C00-3077-154 (MF-93), 1978.

12. K. V. Roberts, J. P. Christiansen, and A. W. Long, Comput . Phys . Commun. 10. $264(1975)$.

13. Y. P. Pao, Phys. Fluids 19, 1177 (1976).

14. R. B. White, D. A. Monticello, M. N. Rosenbluth, and B. V. Waddell, Phys. Fluids 20, 800 (1977).

15. J.'Reid and E. W. Laing, J. Plasme Phys. 21, 501 (1979).

16. J. Reid and E. W. Laing, J. Plasma Phys. 22, 157 (1979).

17. J. Reid and E. W. Laing, J. Plasma Phys. 22. 193 (1979).

18. J. Hogan, Nucl. Fusion 19, 753 (1979).

19. H. Grad, "Alternating Dimension Plasma Transport in Three Dimensions," Courant Institute of Mathematical Sciences report Co0-3077-164 (MF-95), 1979; also in Proc. of the Fourth International Symposium on computing Methods in Rhysics Applied Sciences, and Engineering, R. Glowinski and J. Lions, Eds. (North Holland Publishing Co., 1980).

20. H. Grad, Ann. of the N. Y. Acad. of Sci.. 357, 233 (1980).

21. G. V. Pereversev, V. D. Shafranov, and L. E. Zakharov, in Theoretical and Computational Plosme Phrsics, p. 469 (International Atomic Energy Agency. Vienna, 1978). 
22. V. D. Shafranov, Reviews of Plasmo Physics, Vol.2, p. 103 (M. A. Leontovich, Ed., Consultants Bureau, New York, 1966).

23. M. D. Kruskal and R. M. Kulsrud, Phys. Fluids 1, 265 (1958).

24. A. H. Boozer, Phys. Fluids 24, 1999 (1981).

25. O. Betancourt and P. Garabedian, Proc. Nat. Acad. Sci. USA 73, 984 (1976) .

26. F. Bauer, O. Betancourt, and P. Garabedian, A Computational Method in Plasma Physics (Springer-Verlag. New York, 1978).

27. P. H. Rutherford, Phys. Fluids 16, 1903 (1973).

28. G. Miller, "Error Magnetic fields in a Cylindrical Plasma: Stability With Zero Pressure," LA-UR-84-65 (submitted for publication).

29. J. B. Taylor, Phys. Rev. Lett. 133, 1139 (1974).

30. J. B. Taylor, in Pulsed High Beta Plasmas, D. Evans, Ed. (Pergamon, Oxford, 1976), p. 59 .

31. M. K. Bevir and J. W. Gray in "Proc. of the Reversed Field Pinch Theory Workshop," Los Alamos report LA-8944-LC, 176 (1981).

32. M. K. Bevir, C. G. Gimblett, and G. Miller, "Quasi-Steady State Toroidal Discharges." (submitted for publication).

33. B. B. Kadomstev, in Plasma Physics and Controlled Nuclear Fusion Research 1976. Vol. I, p. 555 (International Atomic Energy Agency, Vienna, 1977).

34. A. Y. Aydemir and D. C. Barnes, Phys. Rev. Lett. 52, 930 (1984).

35. H. K. Moffatt, J. Fluid Mech. 35, 117 (1969).

36. A. Reiman and A. Boozer, Phys. Fluids 26, 1292 (1983).

37. M. G. Rusbridge, Nucl. Fusion 222, 1291 (1982).

38. G. Miller, "A Model of Energy Confinement in the Reversed Field Pinch," Los Alamos report LA-9520-MS, 1982 (to be published in Plasma Phys.).

39. G. Miller, "Steady-State Discharges," LA-UR-83-2688 (submitted for publication). 